\title{
Progress Towards the Completion of the MICE Demonstration of Sustainable Ionization Cooling
}

\author{
Pierrick Hanlet, for the MICE collaboration* $\dagger$ \\ Illinois Institute of Technology, Chicago, IL, USA 60616 \\ E-mail: hanletefnal.gov
}

\begin{abstract}
The Muon Ionization Cooling Experiment (MICE) at the Rutherford Appleton Laboratory aims at demonstrating a $\sim 5 \%$ ionization cooling effect on a muon beam by its interaction with low- $Z$ absorber materials followed by restoration of longitudinal momentum in RF cavities. The final configuration of MICE will add to the Step IV hardware a second Focus Coil module and two 201.25 MHz RF cavities to provide a 10.3 MV/m accelerating gradient. This requires an RF drive system and distribution network to deliver $\sim 2 \mathrm{MW}$ of power in $1 \mathrm{~ms}$ pulses at $1 \mathrm{~Hz}$ repetition rate to each cavity with correct RF phasing and diagnostics to determine the gradient and the muon transit phase. Progress towards the completion of the final configuration is described.
\end{abstract}

16th International Workshop on Neutrino Factories and Future Neutrino Beam Facilities - NUFACT2014, 25 -30 August, 2014

University of Glasgow, United Kingdom

\footnotetext{
${ }^{*}$ Speaker.

${ }^{\dagger}$ work supported by U.S. Department of Energy Office of Science and the National Science Foundation.
} 


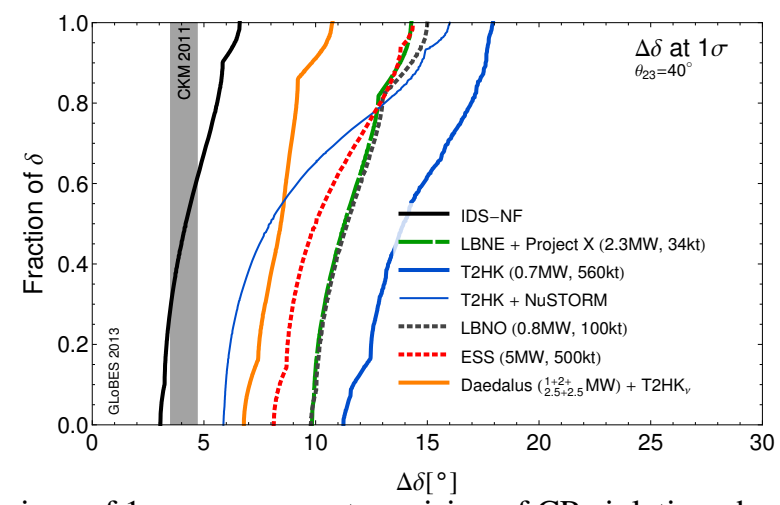

Figure 1: IDS-NF comparison of $1 \sigma$ measurement precision of CP violating phase $\delta$ for different facilities.

\section{Motivation}

The decays $\left(\mu^{-} \rightarrow e^{-} v_{\mu} \bar{v}_{e}\right.$ and $\mu^{+} \rightarrow e^{+} \bar{v}_{\mu} v_{e}$ ) of stored muons in a high intensity Neutrino Factory produce a narrow beam of neutrinos which are fully characterized. The neutrinos are ideal for the study of the "Golden" oscillation channels [1] $\left(v_{e} \rightarrow v_{\mu}\right.$ and $\left.\bar{v}_{e} \rightarrow \bar{v}_{\mu}\right)$ where the sign of the detected muon is opposite to that in the storage ring and for the "Platinum" oscillation channels [1] $\left(v_{\mu} \rightarrow v_{e}\right.$ and $\left.\bar{v}_{\mu} \rightarrow \bar{v}_{e}\right)$. Furthermore with $10^{21}$ muon decays per year, this uniquely clean source of neutrinos would enable the study of CP violation in the lepton sector, thus providing the best chance of its discovery [2, 3, 4]. For example, the precision with which the CP phase $\delta$ can be measured at the IDS Neutrino Factory [1] in comparison to other future facilities is shown in Fig. 1.

Muons for a neutrino factory ${ }^{1}[5,6]$ are produced as tertiary particles $(p+N \rightarrow \pi+X$ with subsequent decay $\pi \rightarrow \mu v$ ), and hence have too large an inherent emittance (beam volume in the $6 \mathrm{D}$ position and momentum phase space) for a cost-effective accelerator. They must therefore be "cooled" to reduce the beam spread both transversely and longitudinally. Due to the short muon lifetime the only feasible technique for muons, which has as yet only been studied in simulations, is ionization cooling. The international Muon Ionization Cooling Experiment (MICE), at the ISIS accelerator at Rutherford Appleton Laboratory (UK), will demonstrate the viability of muon ionization cooling with a variety of beam optics, muon momenta (140-240 MeV/c), and emittances.

MICE is a precision experiment which will measure a $\sim 5 \%$ reduction in beam emittance with a measurement precision of better than $0.1 \mathrm{~mm}$-rad. Thus, it is imperative that the systematic errors be minimized and well understood. For this reason, as well as budget constraints, MICE is staged such that the parameters of the beam, detectors, tracking, and cooling channel components are studied in detail in each step. At present, MICE is in the final stages of building its second stage, for historical reasons named Step IV, which will study the cooling effect of both $\mathrm{LH}_{2}$ and $\mathrm{LiH}$ absorbers for a variety of channel optics settings. The final stage of MICE will include RF cavities to partially restore the longitudinal momentum of the muons and thus demonstrate sustainable ionization cooling.

Normalized beam emittance is given by $\varepsilon_{i, n}=\sigma_{r_{i}} \sigma_{p_{i}} /(m c)$, where $\sigma_{r_{i}}$ and $\sigma_{p_{i}}$ are the $i^{\text {th }}$ component of the RMS spatial and momentum spreads, respectively, and $m c$ is the product of the particle mass and speed of light [5,7]. The normalized emittance accounts for the energy dependence of the beam, since a higher energy beam has smaller geomtrical emittance due to boosting.

\footnotetext{
${ }^{1}$ Also for a muon collider, though cooling requirements are much more demanding and include longitudinal cooling.
} 
In ionization cooling, the muons lose energy traversing a low- $Z$ absorber and have the longitudinal component of momentum restored in accelerating cavities, all while being focused in a magnetic lattice. In traversing the absorber, muons lose momentum in all directions- "cool"while Coulomb scattering increases emittance- "heat". The rate of change of $\varepsilon_{n}$ thus has both a cooling term and a heating term when traversing a path length $s$ :

$$
\frac{d \varepsilon_{n}}{d s} \approx-\frac{1}{\beta^{2}}\left\langle\left|\frac{d E_{\mu}}{d s}\right|\right\rangle \frac{\varepsilon_{n}}{E_{\mu}}+\frac{1}{\beta^{3}} \frac{\beta_{\perp}(13.6 \mathrm{MeV} / \mathrm{c})^{2}}{2 E_{\mu} m_{\mu} X_{0}} .
$$

Here $\beta c$ is the muon velocity, $\left\langle d E_{\mu} / d s\right\rangle$ is the average rate of energy loss, $E_{\mu}$ and $m_{\mu}$ are the muon energy and mass, $\beta_{\perp}$ is the transverse betatron function (focal length) evaluated at the absorber, and $X_{0}$ is the radiation length of the absorber material. Note that heating is reduced by strong focusing in the absorber (low $\beta_{\perp}$ ) and by use of a low- $Z$ absorber to increase $X_{0}$.

\section{MICE Description}

To perform the cooling measurement, MICE must: 1) create a muon beam, 2) identify the particles as muons-reject other particles, 3) measure the muon's position and momentum prior to cooling, 4) tightly focus the muons and cool them in a low- $Z$ absorber, 5) restore the longitudinal component of momentum in RF cavities, 6) re-measure the muon's position and momentum after cooling, and 7) identify muons-reject those events in which the muon decayed.

MICE is a single particle experiment, i.e., we measure the individual muons using particle physics techniques, and combine the individual measurements to form an ensemble used in the emittance measurements. The normalized root mean squared emittance, $\varepsilon_{n}$, is determined from the evaluation of the covariance matrix [8]:

$$
\varepsilon_{n}=\frac{1}{m_{\mu}} \sqrt{|\mathbf{V}|} \quad \text { with matrix elements } \quad \mathbf{V}_{i, j}=\frac{1}{N} \sum_{k=1}^{N}\left(u_{i, k}-\mu_{i}\right)\left(u_{j, k}-\mu_{j}\right)
$$

for an ensemble of $N$ measurements and using the vector $\vec{u}=(\vec{x}, \vec{p})$, where we measure $\vec{x}=(x, y, t)$ and $\vec{p}=\left(p_{x}, p_{y}, E\right)$, and $\mu_{i}$ is the mean value of $u_{i}$. The measured cooling effect is then the before minus after difference between the measured normalized emittances.

Figure 2 shows a schematic of the MICE muon beamline. The muon beam is created using a titanium target that is dipped at $\sim 1 \mathrm{~Hz}$ with acceleration $\sim 90 \mathrm{~g}$ into the ISIS synchrotron beam during the last $3 \mathrm{~ms}$ of the ISIS acceleration cycle. The pions produced in the collision are transported to the MICE Hall and momentum selected using conventional quadrupole triplet (Q1-3) and dipole (D1) magnets. Following D1 is the superconducting Decay Solenoid (DS) which strongly focuses the pions while increasing their path length, thus increasing the total muon flux by an order of magnitude compared to a conventional quadrupole channel. Following the DS, muons are momentum selected and transported to the cooling channel with a dipole (D2) and quad triplets (Q4-6 \& Q7-9). Results of the characterization of the MICE muon beamline can be found in $[9,10]$.

Particle Identification (PID) on the incoming beam is performed with two threshold Cherenkov counters and two time-of-flight scintillator hodoscopes (ToF0 \& ToF1) up and downstream of the last quadrupole triplet as seen in Fig. 2. These detectors serve to identify muons and reject other 

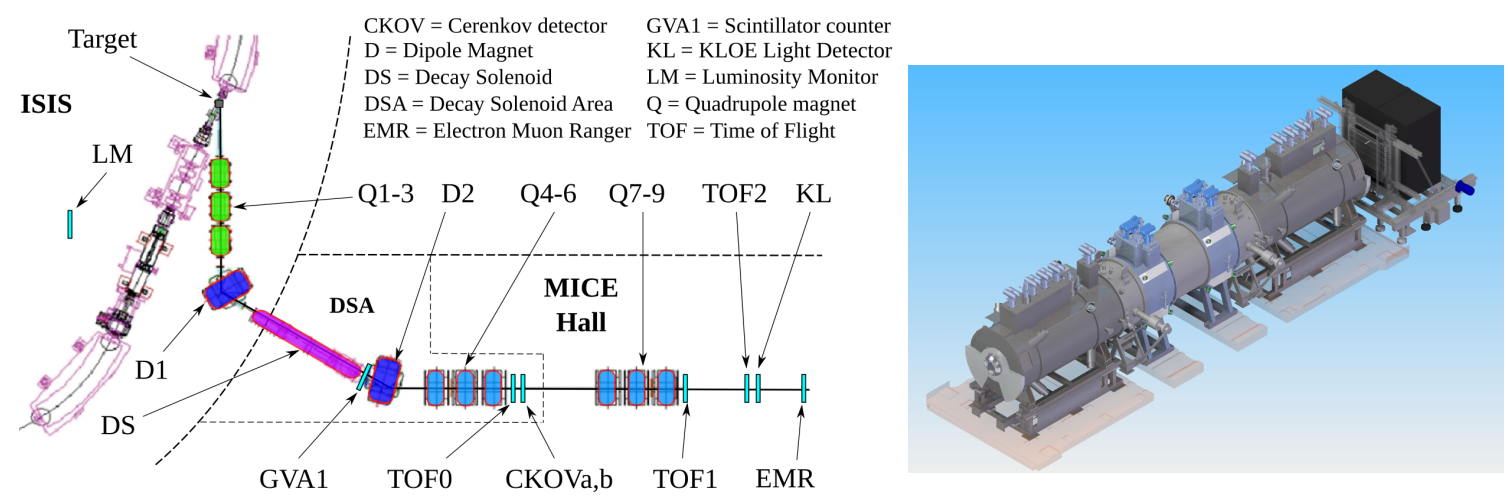

Figure 3: MICE Cooling Channel: Final Figure 2: MICE muon beamline schematic, beginning from the Configuration. Muons enter from the lower target in a section of ISIS. Note that the downstream detectors, left and the EMR is the black box.

ToF2, KL, \& EMR, are downstream of the MICE cooling channel, not shown.

particles. Muons which decay in the channel are rejected using the last ToF plane (ToF2), KLOElight calorimeter (KL), and electron-muon ranger (EMR) downstream of the cooling channel. Data have been collected with all detectors. The ToF detectors were calibrated to have time resolutions of $55 \mathrm{ps}, 53 \mathrm{ps}, 52 \mathrm{ps}$ for ToF0, ToF1, ToF2, respectively [11, 12]. The ToF system is also used as the trigger for the experiment. The beamline and detectors were characterized in the first stage of MICE which was completed in 2011; further details can be found in [10,11].

\section{MICE Step IV}

As of this writing, MICE is entering into the commissioning phase of Step IV. The MICE channel for Step IV consists of an "Absorber/Focus Coil" module (AFC) sandwiched between two identical Tracking Spectrometers (TS), as shown in Figs. 3,4.

A superconducting solenoid magnet, or "Spectrometer Solenoid" (SS), with a fiber tracker in its bore comprises a TS. Each SS cryostat is $2.9 \mathrm{~m}$ in length with a $40 \mathrm{~cm}$ warm bore and has 5 $\mathrm{Nb}-\mathrm{Ti}$ coils wound on a single $6061-T 6 \mathrm{Al}$ mandrel. The longest, $\sim 1.3 \mathrm{~m}$, coil is flanked on either side with trim coils to provide a uniform $4 \mathrm{~T}$ field in the tracking region; the remaining two coils are closest to the AFC and serve to match the beam optics to the cooling channel. Both magnets were built and trained to $102 \%$ of the maximum operating currents at the manufacturer Wang, NMR, Livermore, California, U.S.A. [13]. The tracker is built of 5-planes, 3-120 stereo-view scintillating fibers with $1,400350 \mu \mathrm{m}$ fibers/plane and is positioned coaxially inside the uniform

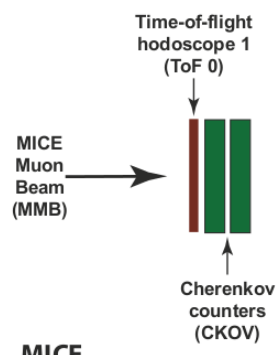

MICE

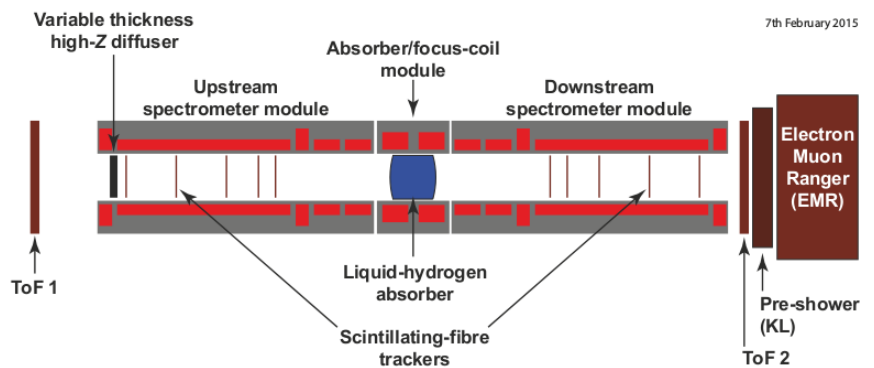

Figure 4: MICE Step IV configuration. 
field. Readout of the trackers is performed with visible light photon counters (VLPC) and D $\varnothing$ AFEIIt electronics $[14,15]$. The tracking spectrometers will be used to measure muon trajectories and momenta, thus providing the inputs to Eq. 2.1 for the emittance measurements.

The Focus Coil (FC) (the "FC" part of the AFC) is also a superconducting solenoidal magnet with $2 \mathrm{Nb}-\mathrm{Ti}$ coils wound on a single $6061-T 6 \mathrm{Al}$ mandrel. The coils can be operated with fields parallel or anti-parallel and provide strong focusing (small $\beta_{\perp}$ in Eq. 1.1) of the muons in the absorber, which is positioned co-axially and longitudinally in the warm bore center of the coils. The magnet for Step IV has been trained to $\sim 1 \%$ above full current in both modes. The absorbers to be studied in Step IV are $\mathrm{LH}_{2}$ and $\mathrm{LiH}$. The cryogenic system for the $\mathrm{H}_{2}$ liquification is installed and has been tested using a dewar, and the absorber body is awaiting installation.

The MICE channel magnets are all built without a magnetic return yoke and thus produce large stray fields which would adversely affect the operation of some of the local electronics. To mitigate this problem, a soft iron partial shell has been designed and is being manufactured to provide a return path for the magnetic flux. It is referred to as the Partial Return Yoke (PRY) and is shown in Fig. 5. Delivery of the PRY is imminent and is the last of the deliverables required for Step IV.

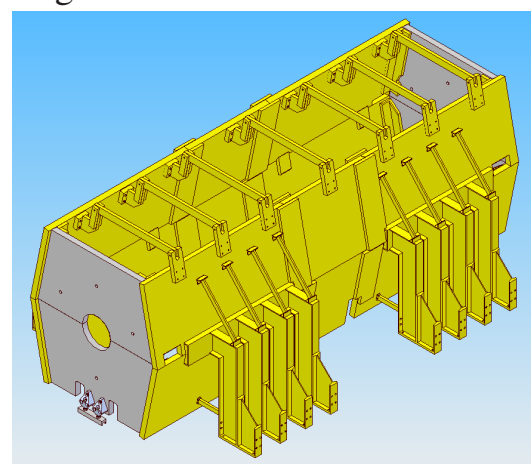

Figure 5: Step IV partial return yoke.

\section{Progress Toward the Final Configuration of MICE}

The major differences between the Step IV configuration and that used in the final configuration-the demonstration of sustainable ionization cooling-is the addition of 2 RF cavities, RF power trains, the second FC, LiH primary and secondary absorbers, spool pieces, and an extension of the PRY to accommodate the longer cooling channel; some of these are shown in Fig. 6. Additionally, because there is no bunching of the muon beam, an accurate phase measurement of each muon with respect to the RF signal in the cavities is required for offline selection of analyzable muons.

\subsection{Hardware Readiness}

Most of the hardware for the MICE final configuration is already in hand. Figure 7 shows one of the 201.25 MHz RF cavities, partially mounted in its "single cavity test stand" (SCTS). Ten such cavities were designed by and built under the direction of LBNL [16, 17]. Also shown in the photo is a newly designed RF coupler arm, and the six "tuner forks" which deform the cavity so as to
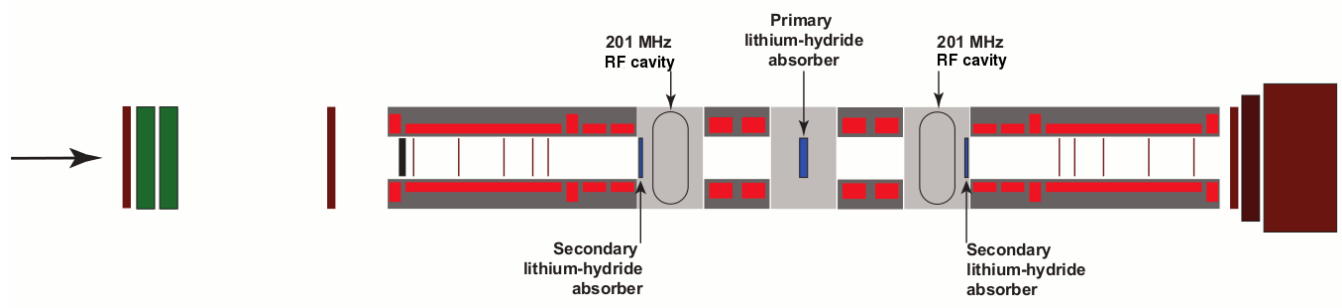

Figure 6: MICE final configuration for the demonstration of sustainable muon ionization cooling. 


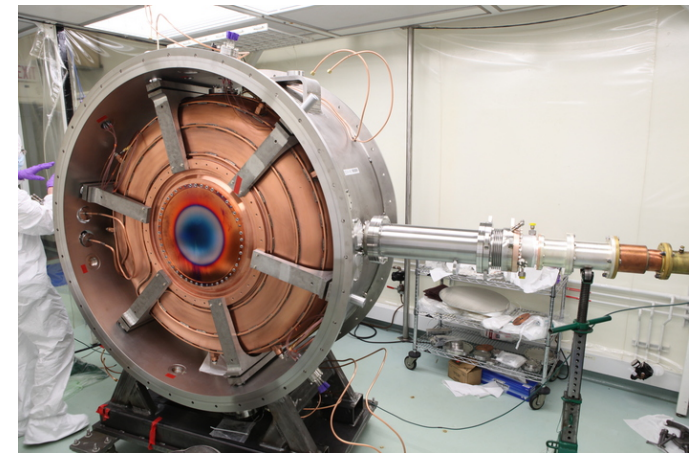

Figure 7: 201.25 MHz RF cavity and coupler.

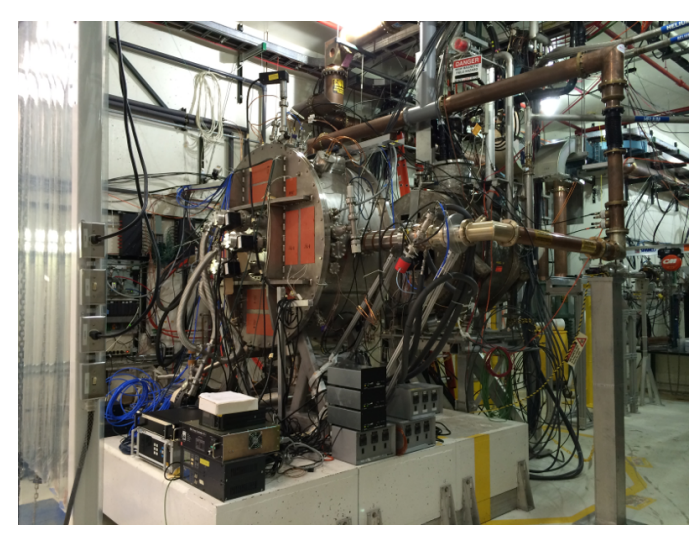

Figure 8: MICE Single Cavity Test Stand in the MuCool Test Area at Fermilab.

dynamically adjust its resonant frequency by $-400 \mathrm{kHz}$ to $+300 \mathrm{kHz}$ [18]. One of the cavities is under test at Fermilab's MuCool Test Area (MTA) [19] and is shown in Fig. 8. Here, the cavity has been operating at its nominal accelerating gradient, $10.3 \mathrm{MV} / \mathrm{m}$, without generating a measurable flux of X-rays or breakdown after $>3 \mathrm{M}$ pulses. The cavity has been tested in the fringe field of the MTA's solenoid magnet; both the magnet and cavity configuration are similar to what the cavity will experience in the fringe field of the MICE FC magnet. In MICE, the cavities will be housed in vacuum vessels essentially identical to the SCTS.

Power to the RF cavities will be provided by a train of a $\sim 4 \mathrm{~kW}$ solid state power amplifier driving a $\sim 250 \mathrm{~kW}$ tetrode driving a $\sim 2 \mathrm{MW}$ triode. [20]. Two such amplifier chains are required to operate the two RF cavities. Each $201.25 \mathrm{MHz}$ chain will be run at $2 \mathrm{MW}$ for $1 \mathrm{~ms}$ pulses at $\sim 1 \mathrm{~Hz}$. One such train has been fully refurbished and has been operated at $2.06 \mathrm{MW}$; the second is currently being refurbished at Daresbury Laboratory, UK.

In the final MICE configuration, as shown in Fig. 6, the absorber will not be positioned within the bore of the FC. Instead, two FCs will straddle the primary LiH absorber. The second FC has also been built and commissioned and meets the requirements for use in the demonstration experiment.

The PRY for the final configuration will simply be an extension of the existing design for Step IV. The steel is presently being procured.

\subsection{Muon Phasing}

The MICE target dips into the ISIS beam during the last 1-3 ms of the ISIS cycle. The produced muons, therefore, go through the MICE channel unbunched and at random times during the MICE "spill". In the final configuration with the addition of RF cavities, only muons that are in the correct phase with the RF wave to be accelerated are of interest for the demonstration of sustainable ionization cooling.

MICE will not perform this selection online, but studies are being performed to optimise our offline measurement of the muon phase with respect to the RF signal. Using the ToF system with $\sim 50 \mathrm{ps}$ temporal resolution and the tracker with its high spatial resolution to project the muons to the first RF cavity, one can determine the RF phase and amplitude when the muon arrives at the cavity. 
We desire $50 \mathrm{ps} / 3=16.7 \mathrm{ps}$ resolution implying $\sim 1^{\circ}$ phase resolution. Since the cavity's linewidth is $\sim 4 \mathrm{kHz}$ in $201.25 \mathrm{MHz}$, i.e. it has a fractional linewidth of $2 \times 10^{-5}$, we expect that the maximum phase shift per cycle is $\sim 0.01^{\circ}$. Since the cavity phase will be recorded every 100 cycles, this results in a projected error of $<1^{\circ}$; this may be substantially improved by the low-level RF (LLRF) feedback loop gain bandwidth.

For analysis, we will require a $1 \%$ amplitude measurement and a $5^{\circ}$ phase measurement as shown graphically in Fig. 9. A $201.25 \mathrm{MHz}$ frequency implies a $4.97 \mathrm{~ns}$ period,

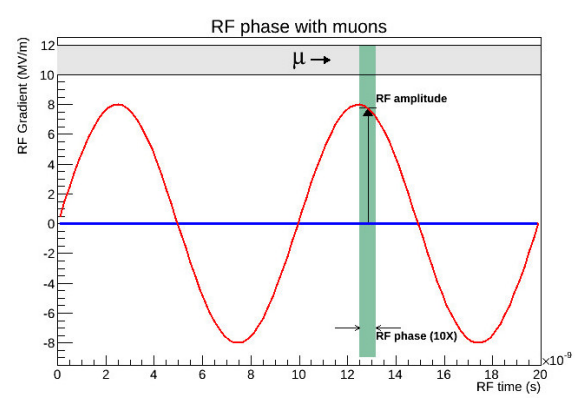

Figure 9: RF phase measurement diagram. where $5^{\circ}$ of $4.97 \mathrm{~ns}$ is $69 \mathrm{ps}$. In both cases RF digitization is required for the amplitude measurement.

\section{Conclusions}

The demonstration of sustainable ionization cooling that MICE will provide is an essential step toward producing and characterizing intense muon beams for a future Neutrino Factory or multi-TeV muon collider.

The MICE demonstration of ionization cooling will be performed using a $\mathrm{LiH}$ absorber and with acceleration provided by two 201.25 MHz cavities. In MICE Step IV, we will study the properties of liquid hydrogen and lithium hydride that affect ionization cooling. In its final configuration, MICE will demonstrate sustainable ionization cooling with moderate additions to the cooling channel which are mostly in hand or in an advanced stage of preparation. The MICE collaboration is on track to deliver its demonstration of ionization cooling by 2017 and so pave the way for a new generation of muon accelerators.

\section{References}

[1] S. Choubey et. al,, "International Design Study for the Neutrino Factory, Interim Design Report", arXiv:1112.2853v1 [hep-ex] (2011), http://www.ids-nf.org.

[2] S. Geer, "Neutrino Beams for Muon Storage Rings: Characteristics and Physics Potential", Phys. Rev. D57 6989 (1998).

[3] A. Bandyopadhyay et al., "Physics at a Future Neutrino Factory and Super-beam Facility", Rept. Prog. Phys. 72 (2009) 106201] (2007).

[4] A. Bross et al., "A Neutrino Factory fo both Large and Small $\theta_{13}$ ”, Phys. Rev. D77 093012 (2008).

[5] C. Ankenbrandt et al., PRST-AB 2, 081001 (1999); M.M. Alsharo'a et al., PRST-AB 6, 081001 (2003).

[6] “Feasibility Study-II of a Muon-Based Neutrino Source," eds. S. Ozaki et al., BNL-52623, http://www.cap.bnl.gov/mumustudyii/FS2-report.html.

[7] D.M. Kaplan, "Introduction to Muon Cooling", arXiv:physics/0109061v3 [physics.acc-ph] (2002). 
[8] C.Rogers and M.Ellis, "High precision measurement of muon beam emittance reduction in MICE", PAC-2005-MPPE013, Prepared for Particle Accelerator Conference (PAC 05), Knoxville, Tennessee, USA, 16-20 May 2005.

[9] D. Adams, et al., "Characterisation of the muon beams for the Muon Ionisation Cooling Experiment", Eur. Phys. J. C73 (2013) 2582.

[10] M. Bogomilov et al., "The MICE Muon Beam on ISIS and the beam-line Instrumentation of the Muon Ionization Cooling Experiment”, J.Inst. 7(2012) P05009.

[11] D. Adams et al., "Characterisation of the muon beams for the Muon Ionization Cooling Experiment", arXiv:1306.1509v2 [physics.acc-ph-ex] (2013).

[12] R. Bertoni et al., Nucl. Instrum. Meth. A615 (2010) 14.

[13] S.P. Virostek et al., "Assembly and Test of a Modified Spectrometer Solenoid for MICE", IPAC-2013-THPME048, International Particle Accelerator Conference (IPAC13), Shanghai, China, 12-17 May 2013, THPME048.

[14] M. Ellis et al., "The Design, construction and performance of the MICE scintillating fibre trackers", Nucl.Instrum.Meth. A659 (2011) 136-153 arXiv:1005.3491 [physics.ins-det] MICE-NOTE-254, FERMILAB-PUB-09-259-APC, IC-PRE-09-01.

[15] P. Rubinov, “AFEII Analog Front End Board Design Specifications”, FERMILAB-TM-2488-PPD (2005).

[16] D. Li et al., "Progress of MICE RFCC Module", IPAC-2012-THPPP093, International Particle Accelerator Conference (IPAC12), New Orleans, Louisiana, USA, 20-15 May 2012, THPPP093 (3954).

[17] T. Luo et al., "Progress on the MICE 201 MHz RF Cavity at LBNL", IPAC-2012-THPPC049, Prepared for International Particle Accelerator Conference (IPAC12), New Orleans, Louisiana, USA, 20-15 May 2012, THPPC049 (3398).

[18] L. Somaschini et al., "Tuner System Simulation and Tests for the $201 \mathrm{MHz}$ MICE Cavity", IPAC-2014-THPRI070, International Particle Accelerator Conference (IPAC14), Dresden, Germany, 15-20 June 2014, THPRI070.

[19] Y. Torun et al., "Assembly and Testing of the First 201-MHz MICE Cavity at Fermilab", NAPAC-2013-WEPMA16, International Particle Accelerator Conference (NA-PAC13), Pasadena, California, USA, 29 September-4 October 2014, WEPMA16.

[20] K. Ronald et al., "The RF System for the MICE Experiment”, IPAC-2013-WEPFI066, International Particle Accelerator Conference (IPAC13), Shanghai, China, 12-17 May 2013, WEPFI066. 